\title{
Index rerum ad Vol. 155
}

Confecit: C. Loeb-Schürch

(B) $=$ Buchbesprechungen - Book reviews - Livres nouveaux (V) = Vortrag - Report -

Communication

Achromatopsia, v. Electro-diagnostic procedures

Acide ascorbique, v. Ascorbic acid

Aderhaut, v. Anterior uveitis, Experimental uveitis, Freund-sches Adjuvans, Oxygen, Uveitis anterior

Albinismus, v. Developmental disorders, Miosis congénilale

Alcohol, v. Pseudo-senile macular degeneration

Amaurosis congenita Leber,

v. Electro-diagnostic procedures

Amblyopia, v. Eleclro-diagnostic procedures, Schielamblyopic

American Ophthalmological Society; Transactions of the American Ophthalmological Society 1966, 477 (B)

Anaphylactic uveitis, v. Experimental uveitis

Angiographie; Гangiographie par soustraction de Гartère ophtal-mique et ses branches, 428 (B)

Angle of the anterior chamber, v. Development

Aniridia, v. Developmental disorders

Anterior chamber, v. Development, Fibrinolysin irrigation

Anterior segment, v. Developmental disorders, Radiation treatment

Anterior uveitis; acute anterior uveitis of unknown origin and its connection with herpes simplex, $300(\mathrm{~V})$

Aqueous humour, v. Glaucome à angle ouvert

Arthritis, v. Freundsches Adjuvans

Ascorbic acid; histochemical localization of ascorbic acid in the ciliary processes of rabbits, 464

Augeninnendruck, v. Developmental disorders, Dystrophia myotonica, Glaucome à angle ouvert, Guanethic'ine

Augenheilkunde; Taschenbuch der Augenheilkunde, 427 (B)

Augenkomplikationen, v. Fibrinolysin irrigation, Kälteschädigung, Phenothiazine-retinopathy, Pseudo-senile macular degeneration

Augenmißbildungen bei einem Kind mit überzähligem Chromosomen-fragment, 390

A- und V-Syndrom, 217

Autoimmunkrankheit, v. Freundsches Adjuvans

Basic Ophthalmology, 476 (B) Behçet's disease, 477 (B) Berichtigung Motilitätsschema, 245

Bindehaut, v. Conjonctivite ligneuse,

Developmental disorders, Lymph-

angiectasia Blépharose moniliforme, v. Urbach-

Wiethe Blickparese, v. Gliomas of the

quadrigeminal plate Book reviews, 427 (B), 476 (B) Buchbesprechungen, 427 (B),476 (B) 
Candida albicans; die experimentelle endogene Entzündung des Augeninnern durch Candida albicans, 476 (B)

Cataracte, v. Cryo-extraction, Developmental disorders, Dystrophia myotonica, Heterohesion, Kerato-conus

Chambre antérieure, v. Development, Fibrinolysin irrigation

Champ visuel, v. Horizontal deviation

Chien, v. Medulloblastoma, Oxygen

Chirurgie, v. Cryo-extraction, Heterohesion

Chlorpromazine, v. Phenothiazine-retinopathy

Index rerum ad Vol. 155

481

Chromosomen, v. Augenmißbildungen

Chromosomenkrankheiten; Augen-befunde beí Chromosomenkrankheiten, 148

Ciliarkörper, v. Ascorbic acid

Clearance, v. Oxygen

Congrès, v. American Ophthal-mological Society, International Council, Ophthalmological

Society

Conjonctivite ligneuse; etude patho-logique, histochimique, patho-génique et thérapeutique de la conjonctivite ligneuse, 169

Conjunctiva, v. Conjonctivite ligneuse, Developmental disorders, Lymphangiectasia

Complications oculaires, v. Fibrino-lysin irrigation, Kälteschädigung, Phenothiazine-retinopathy, Pseudo-senile macular degeneration

Coats' disease, $66(\mathrm{~V})$

Colour vision defects; incidence of known and unknown colour vision defects. A study of 6526 secondary school pupils in Amsterdam, 37 (V)

Congenital nystagmus, v. Electro-diagnostic procedures

Cornea, v. Degeneratio corneae cristallinea, Dégénérescence marginale, Fibrinolysin irrigation, Fluorescin-coloured patterns, Hornhautepitheldystrophie, Kälteschädigung, Keratoconus,

Makroglobulinaemie, Radiation treatment

Cornea World Congress, 427 (B)

Corneal graft, v. Degeneratio corneae cristallinea, Makroglobulinaemie

Corpora quadrigemina, v. Gliomas of the quadrigeminal plate

Corps ciliaire, v. Ascorbic acid

Corps vitré, v. Experimental uveitis, Haemaccel

Cristallin, v. Cryo-extraction, Heterohesion

Cryo-extraction, $56(\mathrm{~V})$

Cryosurgical techniques in ophthalmology; atlas of cryosurgical techniques in ophthalmology,

430 (B)

Cryotherapie, v. Cryoextraction,

Kälteschädigung Cysticercus, v. Ophthalmo-neuro-

cysticercosis

Degeneratio corneae cristallinea hereditaria. A clinical, genetical and histological study, 409

Dégénérescence cornéenne, v. Degeneratio corneae cristallinea, Dégénérescence marginale,

Makroglobulinaemie

Dégénérescence maculaíre, v. Pseudo-senile macular degeneration 
Dégénérescence marginale pellucide de la cornée, 337

Dégénérescence tapéto-rétinienne, v. Dystrophia myotonica, Electro-diagnostic procedures, Keratoconus, Unilateral retinitis pigmentosa

Demonstration of apparatus, $332(\mathrm{~V})$

Deuteranopia, v. Colour vision defects

Development; the development of the angle of the anterior chamber, 330 (V)

Developmental disorders of the anterior ocular segment and xerosis conjunctive, 317 (V)

Diabetes, v. Resistance capillaire

Diagnostik, v. Ultrasonography

Dog, v. Medulloblastoma, Oxygen

Dystrophia corneae, v. Degeneratio corneae cristallinea, Hornhautepitheldystrophie

Dystrophia epithelialis corneae (Typ Meesmann-Wilke) , 81

Dystrophia myotonica; histological findings in a case of dystrophia myotonica, 294 (V)

Dystrophia myotonica; ocular changes in dystrophia myotonica, 291 (V)

EEG, v. Retinal stimuli

Electro-diagnostic procedures; the value of electro-diagnostic procedures (ERG, EOG) in differential diagnosis of congenital nystagmus, 28 (V)

Ophthalmologica, Vol. 155, No. 6 (1968)

34

482

Index rerum

ad Vol. 155

Elect oretinographic threshold value; the difference between the electroretinographic and the sensory threshold values, $78(\mathrm{~V})$

Electroretinography with weak stimuli, 80 (V)

Elektroencephalogramm, v. Retinal stimuli

Elektronenmikroskopie, v. Experimental uveitis

Elektrooculogramm, v. Electro-diagnostic procedures, Pheno-thiazine-retinopathy

Elektroretinogramm, v. ERG

Embryologie, v. Development, Miosis congénitale

Endokrine Ophthalmopathie, 477 (B)

Endothélium cornéen, v. Kälte-schädigung

EOG, v. Electro-diagnostic procedures, Phenothiazine-retinopathy

Epithelial corneal dystrophy of Meesmann-Wilke, 81

Epithelium pigmentaire, v. Dys-trophia myotonica, Experimental uveitis, Miosis congénitale

ERG, v. Electro-diagnostic procedures, Electroretinographic threshold value,

Electroretinography, Retinal stimuli, Unilateral reti-nitis pigmentosa

Experimental uveitis; an electron microscopic study of the earliest changes of the retina in

experimental uveitis, 117

Experimentelle Forschung, v. Ascorbic acid, Experimental uveitis, Freundsches Adjuvans,

Hetero-hesion, Oxygen

Experimentelle Uveitis, v. Freundsches Adjuvans

External diseases of the eye; atlas of external diseases of the eye, 430 (B)

Farbsinn, v. Colour vision defects Fibrinolysin irrigation in traumatic 
secondary hyphema, 357 Fluorescin-coloured polygonal patterns in the normal human cornea and the reflectographic 'Furchen-bild' of Fischer, 299 (V)

Fortbildung und Orientierung, 148

Freundsches Adjuvans; experimentelle Uveitis bei der Ratte nach subkutaner Injektion von

Freundschen Adjuvans. Histo-logische Veränderungen der Uvea, 271

Furchenbild, v. Fluorescin-coloured patterns

Further studies and other information, 148

Gaze palsy, v. Gliomas of the quadri-geminal plate

Gefäße, v. Resistance capillaire

Genetics, v. Chromosomenkrankhei-ten, Colour vision defects, De-generatio corneae cristallinea,

Developmental disorders, Dys-trophia myotonica, Hornhaut-epitheldystrophie, Miosis

congénitale, Oguchi's disease, Urbach-Wiethe

Gesichtsfeld, v. Horizontal deviation

Glaskörper, v. Experimental uveitis, Haemaccel

Glaucome à angle ouvert; etude simultanée de la pression oculaire et de la resistance à

Гécoulement de Thumeur aqueuse aux dif-férents stades de revolution du glaucome à angle

ouvert, 1

Glaucome secondaire, v. Fibrinolysin irrigation

Glaukom, v. Glaucome à angle ouvert, Guanethidine

Gliomas of the quadrigeminal plate and eye movements, 249

Glutenextraction, v. Heterohesion

Greffe cornéenne, v. Degeneratio corneae cristallinea, Makro-globulinaemie

Guanethidine (Ismelin) in the therapy of glaucoma, 194

Haemaccel ${ }^{\circledR}$ as a vitreous substitute,

36 (V) Hemeralopia, v. Electro-diagnostic

procedures

Index rerum

ad Vol. 155

483

Hérédité, v. Chromosomenkrankhei-ten, Colour vision defects, Degene-ratio corneae cristallinea, Developmental disorders, Dystrophia myo-tonica, Hornhautepitheldystrophie, Miosis

congénitale, Oguchi's disease, Urbach-Wiethe

Herpes simplex, v. Anterior uveitis, Radiation treatment

Heterohesion; lens extraction by heterohesion, 141

Histochemie, v. Ascorbic acid, Con-jonctivite ligneuse, Degeneratio corneae cristallinea,

Dégéné-rescence marginals

Histologie, v. Conjonctivite ligneuse, Degeneratio corneae cristallinea, Dégénérescence

marginale, Development, Dystrophia myotonica, Experimental uveitis, Freund-sches Adjuvans,

Gliomas of the quadrigeminal plate, Kälte-schädigung, L·ymphangiectasia,

Makroglobulinaemie, Medullo-blastoma, Miosis congénitale, Sarcoma, Urbach-Wiethe

Horizontal deviation of squint; limitation of suppression to one half of the visual field in the

pathogenesis of the horizontal deviation of squint, $297(\mathrm{~V})$

Hornhaut, v. Degeneratio corneae cristallinea, Dégénérescence marginale, Fibrinolysin

irrigation, Fluorescin-coloured patterns, Hornhautepitheldystrophie, Kälte-schädigung,

Keratoconus, Makroglobulinaemie, Radiation treatment 
Hornhautdegeneration, v. Degeneratio corneae cristallinea, Dégénérescence marginale,

Makroglobulinaemie

Hornhautepitheldystrophie; liber die Variationsbreite der hereditären Hornhautepitheldystrophie

(Typ Meesmann-Wilke) , 81

Humeur aqueuse, v. Glaucome à angle ouvert

Hund, v. Medulloblastoma, Oxygen

Hyalinose, v. Conjonctivite ligneuse

Hyalinosis cutis et mucosae, v. Urbach-Wiethe

Hydrophthalmus, v. Developmental

disorders Hyphaema, v. Fibrinolysin irrigation Hypothermie, v. Kälteschädigung

Immunoreaktion, v. Freundsches Adjuvans

Instrument, v. Heterohesion, Tono-metry, dynamic

International Council of Ophthalmology; Meeting of the International Council of Ophthalmology and of the International Federation of Ophthalmological Societies (1966), 167

Intoxication, v. Phenothiazine-retinopathy, Pseudo-senile macu-lar degeneration

Intraoculardruck, v. Developmental disorders, Dystrophia myotonica, Glaucome à angle ouvert,

Guanethidine

Iridocyclitís, v. Anterior uveitis

Iris, v. Miosis congénitale

Ismelin, v. Guanethidine

Kälteschädigung; zur Kälteschädigung des Hornhautendothels. Untersuchungen am Häutchenpräparat, 87

Kammerwasser, v. Glaucome à angle ouvert

Kammerwinkel, v. Development

Kaninchen, v. Ascorbic acid, Experimental uveitis, Haemaccel, Heterohesion, Kälteschädigung

Kapillarresistenz, v. Resistance capillaire

Katarakt, v. Cryo-extraction, Developmental disorders, Dystrophia myotonica, Heterohesion,

Keratoconus

Keratectomy; atlas of keratectomy and keratoplasty, 429 (B)

Keratoconus in congenital diffuse tapetoretinal degeneration, 8

Keratoconus, v. Dégénérescence marginale

Keratoplastik, v. Degeneratio corneae cristallinea, Makroglobulinaemie

Kongresse, v. American Ophthalmological Society, International Council, Ophthalmological

Society

484

Index rerum

ad Vol. 155

Kryotherapie, v. Cryo-extraction, Kälteschädigung

Lapin, v. Ascorbic acid, Experimental uveitis, Haemaccel, Hetero-hesion, Kälteschädigung

Lens; symposium on the lens, 428 (B)

Lens, v. Cryo-extraction, Hetero-hesion

Libri, 427 (B), 476 (B)

Ligneous conjunctivitis, v. Con-jonctivite ligneuse

Linse, v. Cryo-extraction, Hetero-hesion

Lipo-glycoproteinosis, v. Urbach-Wiethe 
Livres nouveaux, 427 (B), 476 (B)

Low luminances; performance of the eye at low luminances, 429 (B)

Lymphangiectasia; haemorrhagic lymphangiectasia of the conjonc-tiva, 308 (V)

Macula-Degeneration, v. Pseudo-senile macular degeneration

Makroglobulinaemie Waldenström; diffuse Hornhautdegeneration bei einem Fall von

Makroglobulinaemie Waldenström, 449

Malformations, v. Augenmißbildun-gen, Chromoäomenkrankheiten, Developmental disorders,

Kerato-conus, Miosis congénitale

Medulloblastoma in dogs, 77 (V)

Microscopie électronique, v. Experimental uveitis

Miosis congénitale; la miosis congénitale et Гalbinisme, 399

Mißbildungen, v. Augenmißbildun-gen, Chromosomenkrankheiten, Developmental disorders,

Kerato-conus, Miosis congénitale

Moniliforme Blepharose, v. Urbach-Wiethe

Motilität, v. Gliomas of the quadri-geminal plate

Motilitätsschema, Berichtigung, 245

Mycobacterium tuberculosis, v. Freundsches Adjuvans

Myotonische Dystrophie, v. Dys-trophia myotonica

Nachruf, 248, 479

Nécrologe, 248, 479

Netherlands Ophthalmological Society, 156th Meeting (1965), 17-80 (V), 157th Meeting (1966), 291-333 (V)

Netzhaut, v. Coats' disease, Dys-

trophia myotonica, Experimental uveitis, Haemaccel, Keratoconus, Oguchi's disease, Oxygen, Pheno-thiazine-retinopathy, Pseudo-senile macular degeneration, Resistance capillaire,

Unilateral retinitis pigmentosa

Neuro-ophthalmology, v. Gliomas of the quadrigeminal plate, Medulloblastoma, Ophthalmoneuro-cysticercosis

Nystagmus, v. Electro-diagnostic procedures

Obituary, 248, 479

Occhio; Гocchio. Iconografia anato-mica, 428 (B)

Ocular tension, v. Developmental disorders, Dystrophia myotonica, Glaucome à angle ouvert,

Guanethidine

Oguchi's disease, $63(\mathrm{~V})$

Open angle glaucoma, v. Glaucome à angle ouvert

Operation, v. Cryo-extraction, Heterohesion

Ophthalmological Society; Netherlands Ophthalmological Society, 156th Meeting (1965), 17-80

(V), 157th Meeting (1966), 291-333 (V)

Ophthalmological Society of the United Kingdom, 87 th Annual Congress, 1967, 334 (V)

Ophthalmo-neurocysticercosis; a case of associated ophthalmo-neurocysticercosis. Anatomo-

clinical considerations, 379

Oxygen; uveal extraction and clearance of oxygen in normal, inflamed and hyper thermic eyes.

2. Whole blood perfusion of isolated organ and in vivo canine studies, compared, 205 Index rerum

ad Vol. 155

485 
Paralysie du regard, v. Gliomas of

the quadrigeminal plate Paraproteinaemie, v. Makroglobulin-

aemie Parasites, v. Ophthalmo-neuro-

cysticercosis Perfectionnement et orientation, 148 Phenothiazine-retinopathy, 70 (V) Phthisis

bulbi, v. Sarcoma Pigmentdegeneration, v. Phenothiazine-retinopathy Pigmentepithel, v.

Dystrophia myo-

tonica, Experimental uveitis,

Miosis congénitale Pression oculaire, v. Developmental

disorders, Dystrophia myotonica,

Glaucome à angle ouvert,

Guanethidine Prismes, v. Syndrome A et V Protanopia, v. Colour vision defects Pseudo-senile

macular degeneration,

$67(\mathrm{~V})$

Quadrigeminal plate, v. Gliomas of the quadrigeminal plate

Rabbit, v. Ascorbic acid, Experimental uveitis, Haemaccel, Hetero-hesion, Kälteschädígung

Radiation treatment; prospects for radiation treatment of herpetic and other affections of the

anterior segment of the eye, 130

Radiotherapie, v. Radiation treatment

Rat, v. Freundsches Adjuvans

Ratte, v. Freundsches Adjuvans

Recherches expérimentales, v. Ascorbic acid, Experimental uveitis, Freundsches Adjuvans,

Hetero-hesion, Oxygen

Resistance capillaire et rétinopathie diabétique. Effet de ГEtamsylate (Cyclonamine ou

Dicynone), 186

Retina, v. Coats' disease, Dystrophia myotonica, Experimental uveitis, Haemaccel, Keratoconus, Oguchi's disease, Oxygen, Phenothiazine-retinopathy, Pseudo-seni $1 \frac{1}{8}$ macular degeneration,

Resistance capillaire, Unilateral retinitis pig-mentosa

Retinal stimuli; relation between localized retinal stimuli and the visual evoked response in man,

$17(\mathrm{~V})$

Retinitis, v. Coats' disease

Retinitis pigmentosa, v. Unilateral retinitis pigmentosa

Retinopathia diabetica, v. Resistance capillaire

Rhodopsin, v. Oguchi's disease

Sarcoma in an atrophic eye, 333 (V) Schielamblyopie; zur Häufigkeit deГ

Schielamblyopie. Statistische Er-

hebungen an vorschulpflichtigen

Kindern einer städtischen Bevöl-

kerung, 234 Schielen, v. Horizontal deviation,

Schielamblyopie, Syndrome A et V Sedan, Jean, Nécrologe, 479 Segment antérieur, v.

Developmental

disorders, Radiation treatment Sehpurpur, v. Oguchi's disease Sekundärglaukom, v. Fibrinolysin

irrigation Sense of colour, v. Colour vision

defects Sklera, v. Developmental disorders 'Snellen-MedaГ; presentation of the

'Snellen-MedaГ to Dr. J. Schappert-

Kimmijser, 80 (V) Societies, v. American Ophthalmolog- 
ical Society, International Council,

Ophthalmological Society Spongioblastoma polare, v. Gliomas

of the quadrigeminal plate Squint, v. Horizontal deviation,

Schielamblyopie, Syndrome A et V Stereoscope for near vision, 332 (V) Stereotaxic atlas of the forebraín of

the guinea pig. Stereotaktischer

Atlas des Vorderhirns des Meer-

schweinchens. Atlas stéréotaxique

du cerveau antérieur du cobaye.

Atlas estereotáxico del cerebro

anterior del cobaya, 430 (B) Stimulation by light, v. Retinal stimuli Strabisme, v. Horizontal deviation,

Schielamblyopie, Syndrome A et V Suppression, v. Horizontal deviation

of squint Surgery, v. Cryo-extraction, Hetero-

hesion

486

Index rerum

ad Vol. 155

Syndrome A et V; les syndromes A et V, 217

Syndrome, v. Augenmißbildungen, Chromosomenkrankheiten, Coats' disease, Degeneratio corneae cristallinea, Oguchi's disease, Urbach-Wiethe

Tapetoretinal degeneration, v. Dys-trophia myotonia, Electro-diagnostic procedures,

Keratoconus, Unilateral retinitis pigmentosa

Telangiectasis, v. Coats' disease

Tension oculaire, v. Developmental disorders, Dystrophia myotonica, Glaucome à angle ouvert, Guanethidine

Therapie, v. Conjonctivíte ligneuse, Cryo-extraction, Fibrinolysin irrigation, Guanethidine,

Radiation treatment, Syndrome A et V

Thiel, Rudolf, Nachruf, 248

Tonographie, v. Glaucome à angle ouvert

Tonometrie, v. Glaucome à angle ouvert

Tonometry; dynamic tonometry, 332 (V)

Topographie cornéenne; la topo-graphie cornéenne et $\Gamma$ adaptation des lentilles de cornée, 429

(B)

Transactions of the Ophthalmo-logical Societies of the United Kingdom, 428 (B)

Tritanopia, v. Colour vision defects

Tumoren, v. Gliomas of the quadri-geminal plate, Medulloblastoma, Sarcoma

Ultrasonography; some clinical experiences with ultrasonography, 313 (V)

Unilateral retinitis pigmentosa; the 'good' eye in unilateral retinitis pigmentosa, 98

Urbach-Wiethe; manifestations ocu-laires du syndrome d'Urbach-

Wiethe. Hyalinosis cutis et mu-cosae, 433

Uvea, v. Anterior uveitis, Experimental uveitis, Freundsches Ad-juvans, Oxygen, Uveitis

anterior

Uveitis anterior; the incidence of anterior uveitis, 57 (V)

Uveitis, v. Anterior uveitis, Experimental uveitis, Freundsches Ad-juvans, Uveitis anterior 
Vaisseaux, v. Resistance capillaire

Varia, 167, 245, 334 (V), 431, 478

Vascular disorders of the eye; symposium on vascular disorders of the eye. Basic considerations in anatomy and physiology, 427 (B)

Vererbung, v. Chromosomenkrankheiten, Colour vision defects, Degeneratio corneae

cristallinea, Developmental disorders, DystroDhia myotonica, Hornhautepiíheldys-trophie,

Miosis congénitale, Oguchi's disease, Urbach-Wiethe

Vergiftung, v. Phenothiazine-retino-pathy, Pseudo-senile macular degeneration

Vermouth, v. Pseudo-senile macular degeneration

Vessels, v. Resistance capillaire

Visual evoked response, v. Retinal stimuli

Visual field, v. Horizontal deviation

Vitamin C, v. Ascorbic acid

Vitreous, v. Experimental uveitis, Haemaccel

Vordere Bulbusabschnitte, v. Developmental disorders, Radiation treatment

Xerosis conjunctivae, v. Developmental disorders V. Vorderkammer

v. Development

Fibrinolysin irrigation 